\title{
Integrated Hospital Information System (HIS) Special focus on BIRDEM Hospital (600 Beds)
}

\author{
Jahidul Hasan \\ Information Management Unit (IMU), BADAS, Dhaka, Bangladesh \\ Email: jahid@dab-bd.org
}

Received January 4, 2013; revised February 4, 2013; accepted May 1, 2013

Copyright (C 2013 Jahidul Hasan. This is an open access article distributed under the Creative Commons Attribution License, which permits unrestricted use, distribution, and reproduction in any medium, provided the original work is properly cited.

\begin{abstract}
Introduction: Integrated Hospital Information System (HIS) is vital to decision making and plays a crucial role in the success of the organization. Computerization of the medical records and documentation has resulted in efficient data management and information dissemination for the users. Hospital Information System addresses the entire major functional areas of modern multi-specialty hospitals. The package enables improved patient care, patient safety, efficiency and reduced costs. It provides easy access to critical information, thereby enabling management to make better decisions on time. Aims: The short-term objectives of the on-line computerized system are to reduce costs and improve the accuracy and timeliness of patient care, accounting and administration, record keeping, and management reporting. The long-term goal is to build and maintain patient database for analysis of data to facilitate decision-making process. Methods: To run the system it requires some Hardware \& Database for IT Department. The technique involves Patient Registration System, Finance \& Accounts, Human Resource Management System, Laboratory, Out Patient Management System, and Inpatient Management System. Results: In the mid seventies, a complex was established at Shahbag area in the name of Bangladesh Institute of Research and Rehabilitation in Diabetes, Endocrine and Metabolic Disorders (BIRDEM). From 1982 BIRDEM was designated as the WHO collaborating centre for research on prevention and control of diabetes. It is first of its kind outside Europe. Now from 2000 BIRDEM has successfull developed \& implemented some crucial part of HIS System like Patient Admission \& Billing System, Finanace \& Account System, Human Resourse Management \& Payroll System, Store Inventory \& Procurement Management System, Labrotary Management System, Assets Management System, Radiology \& Imaging Management System. Conclusions: It can thus be seen that deploying IT can help the medical profession in improving its quality of service and thus automatically increasing the preparedness and defensiveness. Of course, it is of vital importance that the software must have the right type of modularity and openness so that it is manageable, maintainable and upgradeable. They can perform the complex task of matching, tabulating, calculating, retrieving, printing and securing the data as required. Well designed, integrated computer system can be a great tool in the hands of the hospital management in improving services, controlling cost, and ensuring optimal utilization of facilities.
\end{abstract}

Keywords: Integrated Hospital Information System (HIS); Bangladesh

\section{Introduction}

Hospital Information Systems can be defined as massive, integrated systems that support the comprehensive information requirements of hospitals, including patient, clinical, ancillary and financial management. Hospitals are extremely complex institutions with large departments and units coordinate care for patients. Hospitals are becoming more reliant on the ability of hospital information system (HIS) to assist in the diagnosis, management and education for better and improved services and practices [1].
Hospital Information System (HIS) is vital to decision making and plays a crucial role in the success of the organization. Computerization of the medical records and documentation has resulted in efficient data management and information dissemination for the users. Managers, clinicians and other healthcare workers can now access the information without delay or errors. Present study reveals, the existing system requires upgradation to meet the requirements of the managers and the clinicians. Participants feel HIS assists in decision making, and medical audit. Participants felt that the existing HIS resulted in longer time for OPD consultation and delay in investiga- 
tion results. Majority of the participants feel that HIS helps in education and research.

Hospital Information System addresses the entire major functional areas of modern multi-specialty hospitals. The package enables improved patient care, patient safety, and efficiency and reduced costs. The system provides the benefits of streamlining operations, enhanced administration and control, improved responses, cost control and improved profitability.

The System is developed to maintain a secured database of patients and business information. This system was planned to address the challenges encountered by healthcare centers such as small \& large hospitals and private clinics [2].

\subsection{Importance}

The importance of the HIS are reduced patient waiting time for appointment, billing and collection of medicine from pharmacy and reduced time for diagnostic result preparation by directly capturing the data from machines. Patient records available in the hospital and can be referred by any consultant any time from any location. On-line statistical data is available for middle and top Management for decision making. Financial data is available at any given point of time. Human resource data available on-line for taking any decision on manpower required. In HIS on-line data is available on revenue, patient statistics, doctor performance, department performance and financial data on budget for Management decision. It also helps to improve patient care, cost control and data security [3].

\subsection{Experiences in Asia}

In Southeast Asia the health information technology market continues to grow as healthcare services are improved and expanded.

- Malaysia-Malaysia's healthcare system is well developed with easy access to both primary and secondary care facilities. Health IT is high on the Government's strategic agenda. Malaysia is the world's third most popular destination for medical tourism and the market is expected to grow by 30 percent per year over the next couple of years.

- Singapore-Singapore has one of the most mature healthcare sectors in Southeast Asia with an efficient well-developed infrastructure, a highly educated population and local technological expertise. A 10 year IT Master Plan has a US\$300 million budget for IT systems. The main goal is to have a nationwide electronic health record system.

- Thailand-three main health insurance schemes act as the main healthcare purchasers. There are plans to develop an electronic health information system to link the systems used by these three schemes. Demand for healthcare services has continued to increase since universal healthcare programme became free for patient visits to the doctor or the hospital. Thailand's healthcare system is struggling to cope.

\subsection{Bangladesh Prospect}

The system is well developed in developed countries but almost absent in developing countries like Bangladesh. Some of the private hospital in Bangladesh has taken initiative but could not operate properly. In this regards BIRDEM as a non-profit socio voluntary medical organization has taken initiative and to some extend become successful. So it is a pioneer study in this field.

\section{Aims}

In a large hospital, which devotes considerable emphasis on patient care? The short time objectives of the computerized system are to reduce costs and improve the accuracy and timeliness of patient care, accounting, administration, record keeping, and management reporting. The long-term goal is to build and maintain patient database for analysis of data to facilitate decision-making process.

This vision to maintain complete paperless and film less patient records, where patient will not carry any papers anytime to come to the hospital was a major challenge for us.

\section{Methods}

To run HIS it requires some hardware, software and database for IT department like two node cluster SERVER with storage device, application SERVER, backup SER VER, server rack, switch RAC, KVM switch, on Line UPS, voltage stabilizer, database(RDMS) with RAC, network cable, network switch, RJ-45 connector, laptop, computer, lager printer, DOT matrix printer, technical Instrument.

\subsection{Technique}

The technique involves some systems like Patient Registration, Patient Appointment, Patient Billing, Finance \& Accounts, Pharmacy Management, Medical Store, Procurement Management, Assets Management, Human Resource Management, Payroll Management, Laboratory Management, Patient Appointment Management, Out Patient Management, Inpatient Management, Nursing Care Management, Accident \& Emergency Management , Kitchen Management, Diet \& Nutrition Management, Library Management, OT Management, Infection Control Management, Social Welfare Management, Engineering Management, Intensive Care Information, Mor- 
tuary Information, Forensic Medicine Information, Security \& Access Control Management Systems.

\subsection{Process Description}

When a patient comes to any health center for medical service, the procedures for providing the patient with the desired service(s) are covered in this module which are described below:

There are two types of patients visits a hospital and healthcare center. One type of patients are registered ones, and another type of patients are those who do not get registered (T_ID) with any center. The process description is provided according to the visits (1st visit and subsequent visits of registered patients, and visit of T_ID patients) to a healthcare center. The visits of patients are described below:

1. First Visit of a Registered Patient

2. Subsequent Visits of a Registered Patient

3. Visit of a T_ID Patient

Detailed process description of the above three categories are given below:

\subsubsection{First Visit of a Registered Patient (Shown in Figure 1)}

Processes involved in maintaining and providing service to a new patient visiting for the first time to a hospital or health center who gets registered with the center are as illustrated below:

The patient at first comes to the reception for registration and hospital information. For the patient willing to be registered, his/her primary information will be put into the system and the system generates a registration number known as CRN (Computer Registration Number) for the patient. This CRN will be used throughout the whole system as the reference ID for that particular patient. The receptionist will consider about the patient whether he/ she is a corporate client (preset categories of patients who get special facilities) or not. These and some other categories of patients may have free registration facility. In these cases, such patients will go directly to the Nurse Station, ignoring the step 3.1.2 \& 3.1.3 (The whole process may be carried out at the Nurse Station in case of increased workload at the reception).

From the reception the patient is sent to the Cash Counter to pay the registration fee.

The person in duty at the Cash Counter will receive registration fee from patient and he/she may come to the reception again.

If the patient wants to avail Social Welfare facility, he/she then is sent to the Social Welfare Department. From Social Welfare Department the patient will come to the Nurse Station.

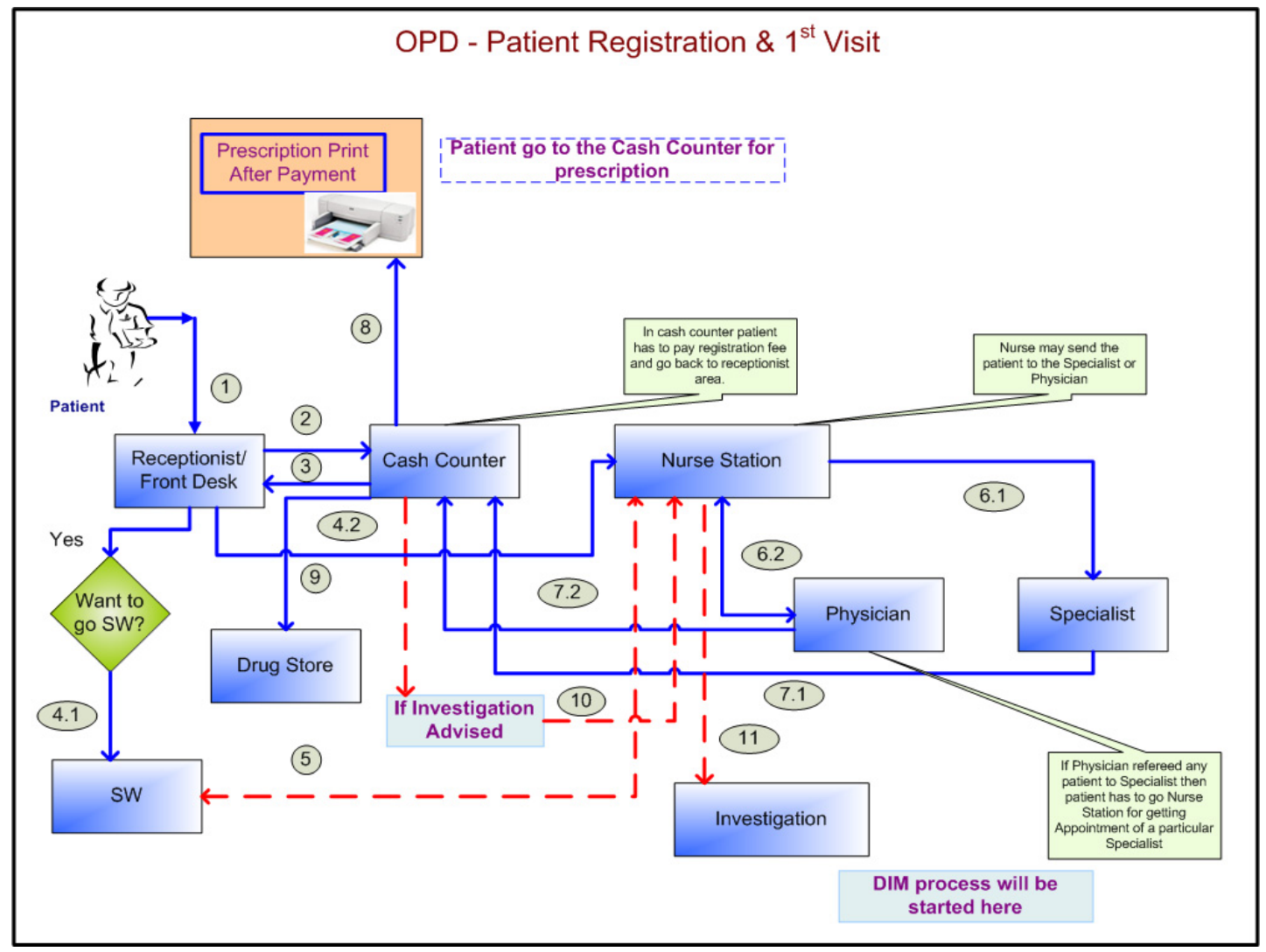

Figure 1. Patient Registration flow for first visit for OPD. 
At the Nurse Station, the patient details his/her complaints the person in duty at the Nurse Station and arranges appointment with the General Physician or with a Specialist and then enters information including Chief Complaints, Past Illness, Family History, Personal History, Anthropometry, General Examination, etc. if necessary.

At the Nurse Station, the patient will again be informed about the Social Welfare facility available for the registered patients.

The patient visits the General Physician and gets his/her advice and treatment. The Physician may advise the patient to see a Specialist for his/her complaints. In this case the patient comes back to the Nurse Station The person in duty at the Nurse Station arranges appointment with the Specialist and the patient follows the process 3.1.6.2.

The patient visits a Specialist and gets his/her advice and treatment.

The patient comes from the Physician / Consultant to the Cash Counter to pay Physician or Consultant's charges. Registered patients are entitled for full or partial payment. They can leave some payments due. From the Cash Counter a prescription will be printed for the patient.
If medicine(s) are prescribed for the patient, and if the patient wants to get the medicines from drug store or pharmacy, the process for taking and/or buying medicine(s) and/or other items will be covered by the Pharmacy module.

If the patient is advised for any investigation by the Physician or the Specialist, the patient comes to the Nurse Station. The person at the Nurse Station may suggest the patient for some available discounts in some investigation(s) and the patient goes to the reception.

From here on, the process of Diagnostic Investigation Management (DIM) module starts.

\subsubsection{Subsequent Visits of a Registered Patient (Shown in Figure 2)}

When a registered patient comes to that hospital or Health care center any time after registration, his/her visit is considered to be the $2^{\text {nd }}$ visit. The procedure to manage such registered patients is illustrated below:

A registered patient at first may come to the reception, or may directly go to the Nurse Station. At the reception the patient is reminded about the Social Welfare facility. If the patient wants to go for Social Welfare, he/she is sent to the Social Welfare department. Else the patient is sent to the Nurse Station.

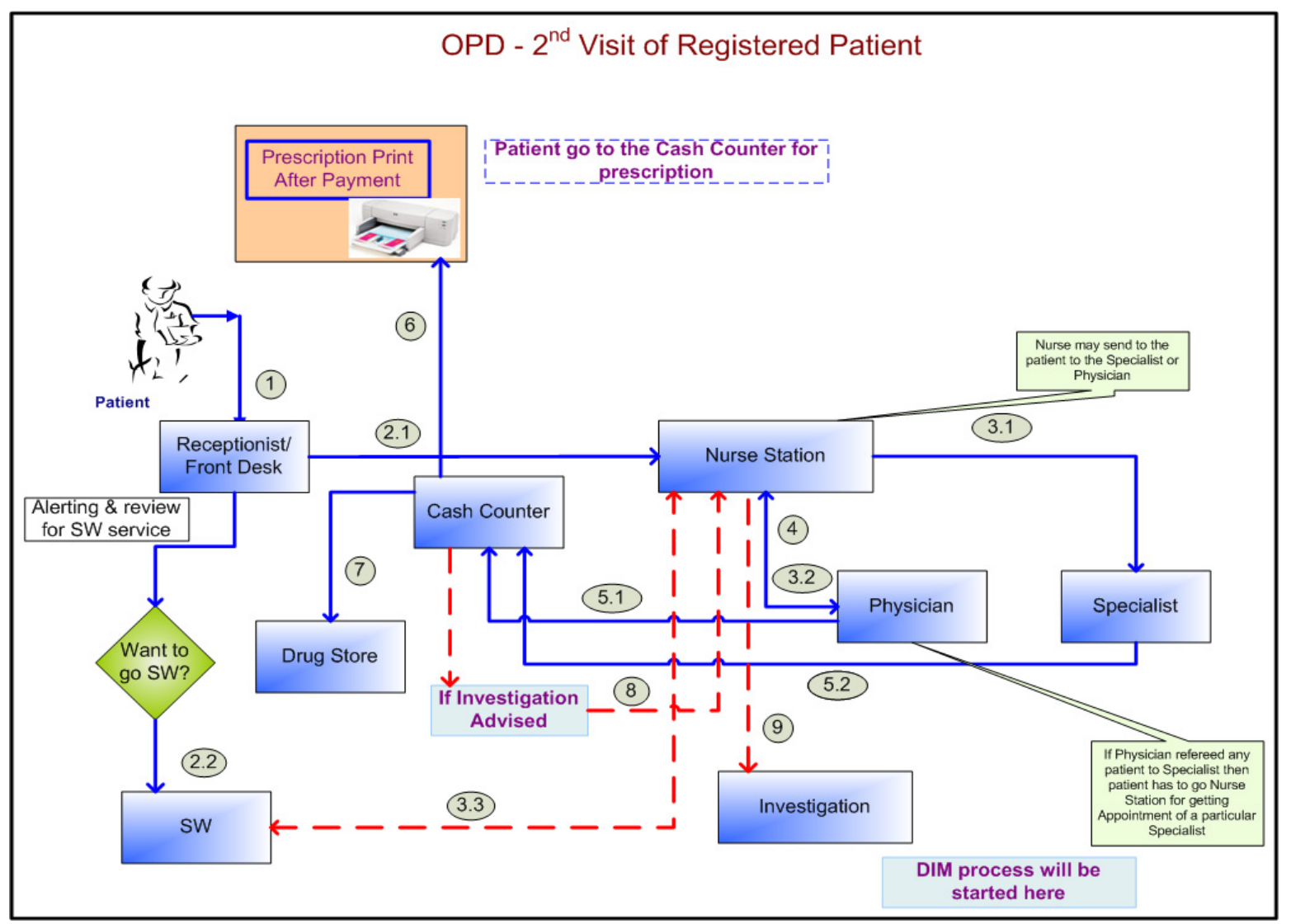

Figure 2. Patient's second visit flow for OPD. 
At the Nurse Station arranges appointment with General Physician or with a Specialist. After appointment is scheduled, the patient details his/her complaints and the person in duty at the Nurse Station enters these information including information like Chief Complaints, Past Illness, Family History, Personal History, Anthropometry, General Examination, etc.

Here the patient is reminded about the availability of the Social Welfare facility and asked if he/she wants to avail the same. If the patient wants to avail Social Welfare facility, he/she then is sent to the Social Welfare Department.

The patient visits a Consultant/Specialist and gets his/her advice and treatment. The Consultant may view/ edit/update the information of the patient's progress sheet like Chief Complaints, Treatment History, Anthropometry, General Examination information entered at the Nurse Station. Information like Systemic Examination, Past Investigation, Provisional Diagnosis, Provisional Treatment, Present Investigation (investigation finding(s) of the immediate past (OPD) visit of the patient, depending on which the Physician may confirm the diagnosis), Confirmed Diagnosis, Confirmatory Treatment, etc. will be entered into the system by the Consultant. (The Specialist may take the print out of the prescription for the patient. Otherwise, it will be done at the Cash Counter).

The patient visits the General Physician and gets his/her advice and treatment. The Physician may view/ edit/update the information of the patient's progress sheet like Chief Complaints, Treatment History, Anthropometry, General Examination information entered at the Nurse Station. Information like Systemic Examination, Past Investigation, Provisional Diagnosis, Provisional Treatment, Present Investigation (investigation finding(s) of the immediate past (OPD) visit of the patient, depending on which the Physician may confirm the diagnosis), Confirmed Diagnosis, Confirmatory Treatment, etc. will be entered into the system by the Physician. (The Physician may take the print out of the prescription for the patient. Otherwise, it will be done at the Cash Counter).

The Physician may refer the patient to a Specialist doctors for his/her complaints. In this case the patient comes back to the Nurse Station The person in duty at the Nurse Station arranges appointment with the Specialist and the patient follows the process 3.2.3.1.

The patient comes from the Physician/Consultant to the Cash Counter to pay Physician or Consultant's charges. Registered patients are entitled for full or partial payment. They can leave some payments due.

From the Cash Counter a prescription will be printed for the patient.

If medicine(s) are prescribed for the patient and if the patient wants to get the medicines from drug store or pharmacy, the process for taking and/or buying medicine(s) and/or other items will be covered by the Phar- macy module.

If the patient is advised for any investigation by the physician or the Specialist, the patient comes to the Nurse Station. The person at the Nurse Station may suggest the patient for some available discounts (in case of corporate patients, and/or discounts assigned for area/ locality, etc.) in some investigation(s) and the patient goes to the reception. From here on, the process of Diagnostic Investigation Management (DIM) module starts.

\subsubsection{Visit of a T_ID Patient (Shown in Figure 3)}

Processes involved in maintaining and providing service to a new patient visiting for the first time to hospital center who is not registered with the center are illustrated below:

The patient at first comes to the reception. The Receptionist enters the primary information of the patient into the system and the system generates a temporary ID number known as T_ID (Temporary ID) for the patient. This T_ID will be used throughout the whole system as the reference ID for that particular patient. The patient is then sent to the Nurse Station

At the Nurse Station, the patient details his/her complaints and the person in duty at the Nurse Station decide whether the patient should visit a General Physician or a Specialist and informs the patient about the consultancy charges. If the patient agrees to go for consultancy, he/she is sent to the Cash Counter to pay the consultancy charges.

The patient pays the consultancy charges at the Cash Counter, receives a payment slip and comes back to the Nurse Station. The person in duty at the Nurse Station arranges appointment with the General Physician or Specialist and enters the patient's data including information like Chief Complaints, Past Illness, Family History, Personal History, Anthropometry, General Examination, etc.

The patient comes to the Physician and receives his/her advice and treatment. The Physician may view/ edit/update the information of the patient's progress sheet like Chief Complaints, Treatment History, Anthropometry, General Examination information entered at the Nurse Station. Information like Systemic Examination, Past Investigation, Provisional Diagnosis, Provisional Treatment, Present Investigation (investigation finding(s) of the immediate past (OPD) visit of the patient, depending on which the physician may confirm the diagnosis), Confirmed Diagnosis, Confirmatory Treatment, etc. will be entered into the system by the Physician. (The Physician may take the print out of the prescription for the patient. Otherwise, it will be done at the Cash Counter).If the physician advises the patient to see a Specialist at any center, and then he/she is sent to the Nurse Station.

The person in duty at the Nurse station arranges appointment with the Specialist and sends the patient to the 


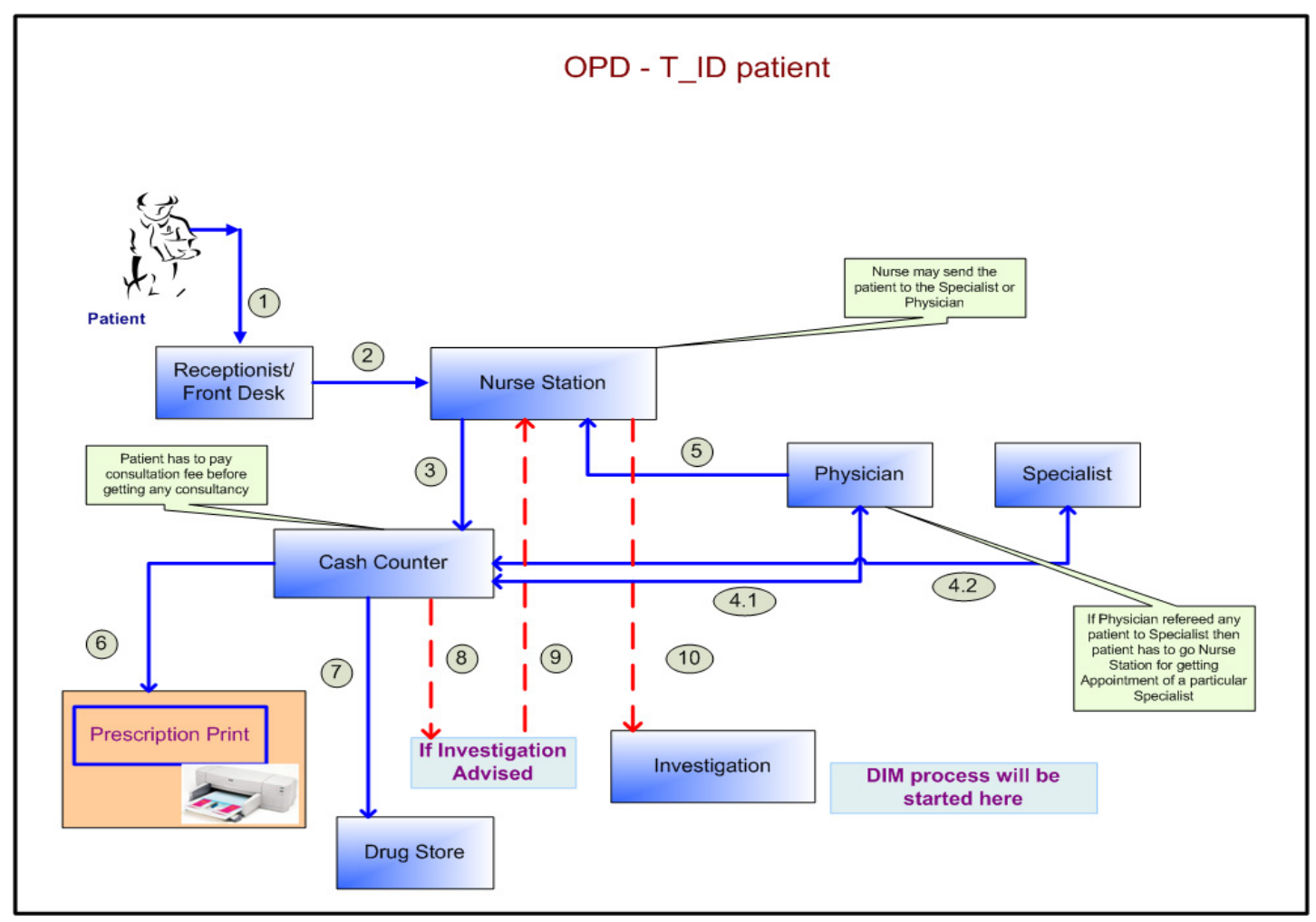

Figure 3. T_ID flow for OPD.

Cash Counter to pay the consultancy charges of that specialist. The T_ID patient has to pay $100 \%$ of the consultancy charges of the Specialist before going to consult with the Specialist. The Consultant may view/edit/update the information of the patient's progress sheet like Chief Complaints, Treatment History, Anthropometry, General Examination information entered at the Nurse Station. Information like Systemic Examination, Past Investigation (investigation finding(s) of the immediate past (OPD) visit of the patient, depending on which the physician may confirm the diagnosis), Provisional Diagnosis, Provisional Treatment, Present Investigation, Confirmed Diagnosis, Confirmatory Treatment, etc. will be entered into the system by the Consultant.

If medicine(s) are prescribed for the patient and if the patient wants to get the medicines drug store or pharmacy, the process for taking and/or buying medicine(s) and/or other items will be covered by the Pharmacy module.

If the patient is advised for any investigation by the Physician or the Specialist, the patient comes to the Nurse Station. The person in duty at the Nurse Station may suggest the patient for some available discounts (in case of corporate patients, and/or discounts assigned for area/locality, etc.) for some investigation(s) and the patient goes to the reception.

From here on, the process of Diagnostic Investigation Management (DIM) module starts.

\section{Results}

\subsection{Success in BIRDEM Hospital}

In the mid seventies Bangladesh Institute of Research and Rehabilitation in Diabetes, Endocrine and Metabolic Disorders (BIRDEM) is established in shabag [4]. The name implies that it was planned from the very beginning that all facilities for academic and research activities will be available within the Association. It is a 600-beded hospital. BIRDEM is now providing diabetic care to almost 0.4 million registered diabetic patients from which about four thousand registered diabetic patients are taking health services from BIRDEM OPD every day. In a small survey it is indicated that the prevalence of diabetes in Bangladesh in populations aged over 15 years is between $2 \%-5 \%$. This type of similarity is also found in most of the under-developed countries. As diabetes may affect other system of the body, BIRDEM adopted a multidisciplinary approach to its services. Gradually, BIRDEM established specialized disciplines like, Cardiology, Gastro-enterology, Surgery, Gynecology and Obstetrics, Nephrology etc. From 1982 BIRDEM was designated as the WHO collaborating centre for research on prevention and control of diabetes, endocrine and metabolic disorders. It is first of its kind outside Europe. Now from 2000 BIRDEM has success full developed \& implemented some crucial part of HIS like Patient Billing System, Finance \& Account System, Human 
Resource Management \& Payroll System, Medical Store \& Procurement Management System, Laboratory Management System, Assets Management System, Radiology \& Imaging Management system, OT Management System etc.

It can thus be seen that deploying some crucial parts of HIS in BIRDEM, improving healthcare facilities, as a result number of indoor and outdoor patient in BIRDEM Hospital has been increased (shown in Figures 4 \& 5).

\subsection{Challenges of HIS}

The main challenges to implementation of HIS in the rural hospitals are lack of computer equipment with high speed internet facilities, lack of computer skill manpower and cost of computers. Therefore from the findings the current ICT technologies in place within the rural areas in developing countries are still unreliable, inaccessible and not sustainable.

- Some peoples some time resist the computerization.

- Shortage of skill technical people in HIS in Bangladesh

- In a hospital most of top label management is doctors, so they can understand the importance of IT in hospital.

- Designing the system to be followed in all the departments in computerized environment

- Designing the software for all the specialty doctors for e-prescriptions and e-medical records from the day one of the starting of the hospital

- Making live from the day of starting the hospital

- Complete electronic patient record

- Fully integrated front office and back office operations

- Also paperless environment for materials, pharmacy, finance and HR

\subsection{Increase Indoor Patients}

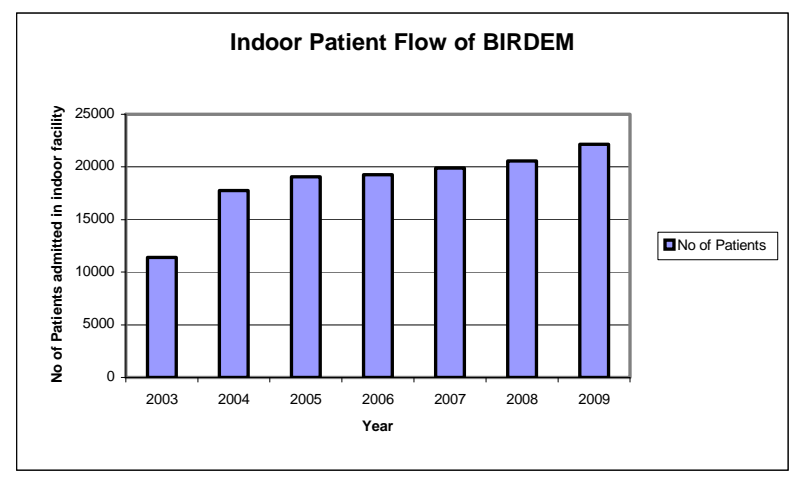

Figure 4. Increase indoor patients.

\subsection{Increase out Door Patient}

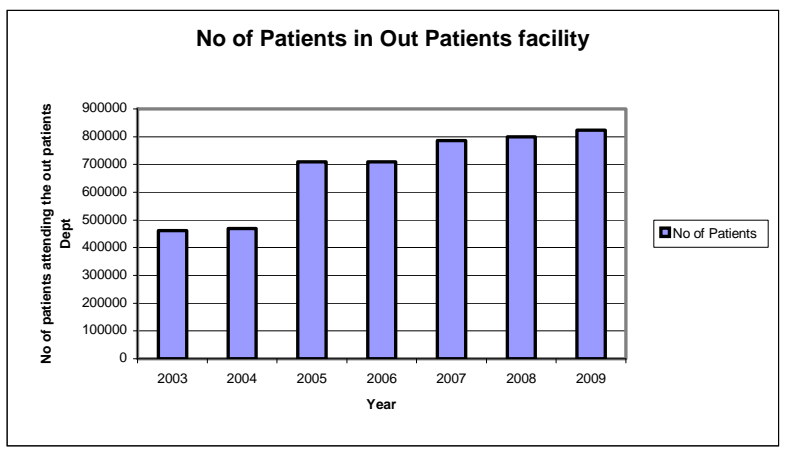

Figure 5. Increase outdoor patients.

\section{Conclusion}

It can thus be seen that deploying IT can help the medical profession in improving its quality of service and thus automatically increasing the preparedness and defensiveness. Of course, it is of vital importance that the software must have the right type of modularity and openness so that it is manageable, maintainable and upgradeable. The hardware should also be reliable, available and have the necessary performance capacity. Certainly, computers with their intrinsic power can play a major role in a hospital. Computers can act as a communication link between departments and allow the common database to be shared by them. They can perform the complex task of matching, tabulating, calculating, retrieving, printing and securing the data as required. Well designed, integrated computer system can be a great tool in the hands of the hospital management in improving services, controlling cost, and ensuring optimal utilization of facilities.

\section{Acknowledgements}

I highly acknowledge the continuous support of Diabetic Association of Bangladesh and its Information Management Unit (IMU).

\section{REFERENCES}

[1] http://en.wikipedia.org/wiki/Hospital_information_syste $\mathrm{m}$

[2] http://khawarsoft.net/PrdHMIS.aspx

[3] http://www.ehow.com/list_6902712_benefits-hospital-ma nagement-information-systems.html

[4] http://www.birdem-bd.org/success.php 